\title{
Researching Multipath TCP Adoption
}

\author{
Henna Warma and Heikki Hämmäinen \\ Aalto University, Department of Communications and Networking, \\ Otakaari 5, 02150 Espoo, Finland \\ \{henna.warma, heikki.hammainen\} @tkk.fi
}

\begin{abstract}
The adoption process of a new Internet protocol or only a change to an existing one is anything but trivial. The classical diffusion theory does not apply as such for studying protocol adoption because the deployment of a protocol usually requires the involvement of multiple stakeholders with varying interests. Multipath TCP (MPTCP) is a new interesting change to the TCP/IP protocol suite which is an extension to regular TCP. MPTCP exploits the idea of resource pooling principle by splitting the data of a single TCP connection across multiple paths in the Internet. The research introduced in this paper aims to identify and evaluate the incentives of different stakeholders to adopt MPTCP. This paper summarizes the proceedings in MPTCP research from the socio-economic point of view and the plans how MPTCP adoption could be studied further. Also, the main problematics of the research are discussed in the paper.
\end{abstract}

Keywords: Future Internet, multipath TCP, adoption incentives.

\section{Introduction}

During the last few decades, the importance of the Internet for the society has increased significantly. The Internet has evolved from its early days to be a backbone of many economical and social activities in the society. The Internet was originally designed for a very different purpose than it is used nowadays. The requirements of the Internet performance as well as scalability have changed a lot and the current Internet architecture as such suits badly for the demands and requirements of the modern society [1]. It is inevitable that changes are needed both to the architecture and protocols.

However, the new technical solutions, e.g., protocols for the future Internet should not be developed without a decent socio-economical control. Because the Internet is a very complex system, the adoption of different technical solutions is not trivial at all. Assessing the viability and durability of new technical solutions already in the early phase of the development may significantly increase the probability that they will get adopted.

The social and commercial control of Internet protocols has, for example, an advantage from Internet Engineering Task Force (IETF) point of view. Since standardization is a very time-consuming activity, the efforts should be focused on those solutions which will interest different stakeholders widely and not just a marginal sector of the market players. This type of research that we are intending to carry out may save IETF for doing groundless work or otherwise help in the standardization work. 


\subsection{Research Topic}

Multipath Transmission Control Protocol (MPTCP) is one of the protocols supporting the future Internet design principles [2]. MPTCP is a manifestation of resource pooling principle [3] and it enables the data packets of a single TCP connection to be split across multiple paths in the network. Thus, MPTCP does not only increase the throughput but also improves the resilience of the network connections. From a technical point of view, the deployment of MPTCP requires a relatively small change to TCP/IP stack and it is upon end-users only. Although MPTCP is simple from the implementation point of view, it may have a significant impact on the value networks in the Internet connectivity market.

Although MPTCP has verified benefits from the technical point of view, it is not self-evident that different stakeholders would like to adopt this protocol. To evaluate the viability of MPTCP it is essential to know what the incentives of different stakeholders are to adopt MPTCP. This is what we want to study in the first place. Studying the MPTCP adoption incentives can also give more insight into dynamics of Internet protocol adoption in general.

\section{State of the Research}

MPTCP has gained outstanding attention within the Internet engineering community recently. However, the attention has been more on the technical side and MPTCP has not been studied in detail from the socio-economic point of view. This section summarizes the results achieved so far and suggests three distinct cases which are planned to be studied in the near future.

\subsection{Achievements}

The initial evaluation of MPTCP has been completed from the social and commercial point of view. The possible deployment scenarios have been outlined and their business opportunities have been discussed in [4]. The paper is a high level analysis of MPTCP deployment and the deployment scenarios should be studied in more detailed to get a concrete idea of the viability of each scenario.

The authors have identified in the paper [4] which stakeholders acting in the Internet connectivity market are affected by the MPTCP deployment. Those stakeholders are end-users, connectivity providers (ISPs), software authors/equipment vendors and router/infrastructure vendors. Costs and benefits for different players are outlined without any deeper analysis but this is a good baseline for the further reseach. One conclusion in the paper is that because the implementation of MPTCP is upon endusers only ISPs cannot prevent the deployment of MPTCP but they can still support and enhance the deployment of MPTCP. However, the adoption incentives of each stakeholder are not tenably justified.

In [4] authors also suggest that deployment of MPTCP would be especially attractive when multihoming capability already exist at the end-user device because no hardware updates are not needed. This is the case with many existing mobile devices and therefore the adoption incentives of this scenario for different stakeholders should be especially studied further. 


\subsection{Planned Research}

The following cases explain how we are going to tackle the research question.

Case 1. In the first case we aim to identify all the different factors which are affecting MPTCP adoption and increase the understanding of dependencies of the different factors. For example, do network effects have any effect on MPTCP adoption and if they do, how do they affect demand? The intention is to build a model which would illustrate the system generally. We also try to evaluate the significance of different factors which could later on help us to concentrate on the most important issues.

Case 2. Identifying adoption incentives of software or equipment vendors is highly interesting because they have the possibility actually implement MPTCP. If they do not see MPTCP as a good investment, it is unlikely that they will deploy the protocol. In the second case, we are going to study a specific business case where one provider controls the software in both end-user devices and content servers. There are multiple examples of this case: Nokia with mobile phones and Ovi and Apple with iPhone and iTunes Store to mention but a few. The main objective is to find out whether implementing MPTCP to its devices is profitable or not for that kind of provider. The reason why we want to investigate specifically incentives of this type of providers is that if the business case is lucrative for them, they could significantly enhance the deployment of MPTCP.

Case 3. In Case 3 we would like to research incentives of ISPs to adopt MPTCP into their business. Although in most scenarios presented in [4] ISPs do not have the ability to implement MPTCP on their own, they can enhance the deployment of MPTCP by offering lucrative service contracts to their customers. The approach would be topdown and we could assume that MPTCP would be deployed. Modeling, for example, different kind of pricing schemes for the MPTCP usage we could find the incentives of an ISP to offer MPTCP related service contracts to its customers.

\subsubsection{Research Methods}

System dynamics is a method for modeling complex systems [6] over time. The different stakeholders affecting of being affected by MPTCP adoption model a complex system which is continuously changing. That is why system dynamics could be also feasible method for modeling the factors affecting MPTCP adoption in Case 1. It would also clarify the relationships of different factors and help to understand the complexities of the research area.

In Case 2, the intention is to use at least functional cost modeling, e.g. [5], to analyze the data. To be able to apply this method we have to identify costs of implementing and running MPTCP exactly. So, the implementation and operational costs should be separated in the model.

\section{Problematics of the Research}

Since the planned research is focusing on the future and MPTCP has not even been implemented yet, it is very difficult to obtain data to be analyzed. However, one good way is to interview some experts from different operators or network equipment vendors but 
still the amount of reliable data may be limited. Therefore, many assumptions have to be made and if the assumptions are not accurate enough, the results may end up being too far from reality which may decrease the credibility and usefulness of the research.

Another problematic issue is how we actually define adoption. This may not be a big problem with the first case but more with the second case. The end-user host, for example, may be MPTCP capable even though the end-user is not using MPTCP. So, the end-user might have the MPTCP capability without making any aware decision to invest on MPTCP. Is MPTCP adopted in this case? This is what we really need to consider before diving deeper into analysis.

Finally, if we think about the problem in a larger scope another issue occurs. Since the research plans presented in this paper are supposed to be the first steps towards a doctorial thesis it would be useful to know how the research cases could be mapped into a bigger scope. It would be much easier to carry out the research if we had in mind what we ultimately want to find out. Because the research is very at the beginning, long-term plans still remain unclear.

\section{References}

1. Handley, M.: Why the Internet Only Just Works. BT Technology Journal 24 (2006)

2. Ford, A., Raiciu, C.: TCP Extensions for Multipath Operation with Multiple Addresses. IETF Internet-Draft (work in progress), draft-ford-mptcp-multiaddressed (July 2009)

3. Wischik, D., Handley, M., Braun, M.B.: The Resource Pooling Principle. ACM SIGCOMM CCR 38(5), 47-52 (2008)

4. Levä, T., Warma, H., Ford, A., Kostopoulos, A., Heinrich, B., Widera, R., Eardley, P.: Business Aspects of Multipath TCP Adoption. In: Future Internet Assembly (FIA) Book, Valencia, Spain (2010)

5. Dilip, J., Chuang, J., Stoica, I.: Modeling the Adoption of new Network Architectures, CoNEXT (2007)

6. Sterman, J.: Business Dynamics - Systems Thinking and Modeling for a Complex World. McGraw-Hill, New York 